\title{
Isoscalar giant resonances for nuclei with mass between 56 and 60
}

\author{
Y.-W. Lui, D. H. Youngblood, H. L. Clark, Y. Tokimoto, and B. John* \\ Cyclotron Institute, Texas A\&M University, College Station, Texas 77843, USA
}

(Received 19 October 2005; published 26 January 2006)

\begin{abstract}
The giant resonance region from $10 \mathrm{MeV}<E_{x}<62 \mathrm{MeV}$ in ${ }^{56} \mathrm{Fe},{ }^{58} \mathrm{Ni}$, and ${ }^{60} \mathrm{Ni}$ has been studied with inelastic scattering of $240 \mathrm{MeV} \alpha$ particles at small angles, including $0^{\circ}$. Most of the expected isoscalar $E 0$ and $E 2$ strength has been identified below $E_{x}=40 \mathrm{MeV}$. Between 56 and $72 \%$ of the isoscalar $E 1$ strength has been located in these nuclei. The mass dependence of the giant monopole energy between $A=40$ and 90 is compared to relativistic and nonrelativistic calculations for interactions with compressibility of nuclear matter $K_{\mathrm{NM}} \sim 211-225 \mathrm{MeV}$.
\end{abstract}

\section{INTRODUCTION}

The location of the isoscalar giant monopole resonance (GMR) and giant dipole resonance (ISGDR) are important because their energies can be directly related to the nuclear compressibility and from this the compressibility of nuclear matter $\left(K_{\mathrm{NM}}\right)$ can be obtained [1,2]. In the past few years, experiments with much improved peak-to-continuum ratio have been performed mostly in heavy nuclei $A \geqslant 90[3,4]$ as well as in light nuclei $A \leqslant 40$ [5-8]. Together with development of the multipole analysis program, high precision strength distributions of isoscalar multipoles in these nuclei have been obtained. With the availability of a large amount of data over a wide range of $A(12 \leqslant A \leqslant 208)$, we have also studied the mass dependence of the GMR $[3,8]$. In heavy nuclei $(A \geqslant$ 110 ), the shape of GMR strength distribution is typically symmetric (Gaussian-like) [9]. In ${ }^{90} \mathrm{Zr}$, the shape changes to mostly symmetric with a tail on the high excitation side of the GMR [3]. In ${ }^{58} \mathrm{Ni}$ and ${ }^{40} \mathrm{Ca}$ the GMR is asymmetric with a slower slope on the high excitation side of the peak and in nuclei with $A \leqslant 28$ the GMR becomes fragmented. The origin of this is not clear; it may be because of nuclear structure or some other effects. From our data, the transition from mostly symmetric to asymmetric shape occurs between ${ }^{90} \mathrm{Zr}$ and ${ }^{58} \mathrm{Ni}[10]$; however, between $40 \leqslant A \leqslant 90$, only ${ }^{58} \mathrm{Ni}$ has been thoroughly studied. It is important to have more information in this region to study this interesting effect and the mass dependence of GMR.

Before 1995, only small amounts of GMR strength had been located in nuclei around $A=60$ [11] using inelastic $\alpha$ scattering with $E_{\alpha} \leqslant 130 \mathrm{MeV}$. Although small amounts of GMR strength had been seen [12] with ${ }^{3} \mathrm{He}$ scattering, the continuum in the ${ }^{3} \mathrm{He}$ spectra was apparently obscuring significant GMR strength in many nuclei [13]. In 2000, using inelastic scattering of $240 \mathrm{MeV} \alpha$ particles at small angles and a folding model analysis, $74 \%$ of the $E 0$ strength was located between $E_{x}=12.0$ and $31.1 \mathrm{MeV}$ in ${ }^{58} \mathrm{Ni}[10]$.

Therefore, we have studied the giant resonance region in ${ }^{56} \mathrm{Fe}$ and ${ }^{60} \mathrm{Ni}$ with small-angle inelastic $\alpha$ scattering at

\footnotetext{
*Present address: Nuclear Physics Division, Bhabha Atomic Research Center, Mumbai 400085, India.
}

$240 \mathrm{MeV}$ to obtain the GMR strength distributions for these nuclei. Because of the excellent peak-to-continuum ratio $[6,8]$ with the $240-\mathrm{MeV} \alpha$ data, the actual distribution of strength between $E_{x}=10$ and $40 \mathrm{MeV}$ can be obtained, even though the cross sections at high excitation in these nuclei are smaller than those in heavy nuclei. As we have developed a much improved multipole analysis program since the analysis reported in Ref. [10], the ${ }^{58} \mathrm{Ni}$ data reported in Ref. [10] have also been reanalyzed.

\section{EXPERIMENTAL TECHNIQUE AND DATA ANALYSIS}

The experimental technique has been described thoroughly in Refs. [6,8] and is summarized briefly below. A $240-\mathrm{MeV}$ $\alpha$-particle beam from the Texas A\&M K500 superconducting cyclotron bombarded self-supporting foils with thicknesses of $12.2 \mathrm{mg} / \mathrm{cm}^{2}\left({ }^{56} \mathrm{Fe}\right)$ and $12.1 \mathrm{mg} / \mathrm{cm}^{2}\left({ }^{60} \mathrm{Ni}\right)$, respectively, located in the target chamber of the multipole-dipole-multipole spectrometer. The horizontal acceptance of the spectrometer was $4^{\circ}$ and ray tracing was used to reconstruct the scattering angle. The vertical acceptance was set at $\pm 2^{\circ}$. The focal plane detector measured the position and angle in the scattering plane and covered energies from $E_{x} \sim 7 \mathrm{MeV}$ to $E_{x} \sim$ $62 \mathrm{MeV}$, depending on the scattering angle. The out-of-plane scattering angle was not measured. A position resolution of approximately $0.9 \mathrm{~mm}$ and a scattering angle resolution of about $0.09^{\circ}$ were obtained. At $\theta_{\text {spec }}=0^{\circ}$, runs with an empty target frame had an $\alpha$-particle rate approximately $1 / 2000$ th of that with a target in place, and $\alpha$ particles were uniformly distributed in the spectrum. Cross sections were obtained from the charge collected, target thickness, dead time, and known solid angle. The target thicknesses were measured by weighing and checked by measuring the energy loss of the $240-\mathrm{MeV}$ $\alpha$ beam in each target. The cumulative uncertainties in target thickness, solid angle, and so on, result in about a $\pm 10 \%$ uncertainty in absolute cross sections. ${ }^{24} \mathrm{Mg}$ spectra were taken before and after each run with each target, and the $13.85 \pm$ $0.02 \mathrm{MeV} L=0$ state [14] was used as a check on the calibration in the giant resonance region.

Data were taken with the spectrometer at $0.0^{\circ}\left(0.0^{\circ}<\theta<\right.$ $\left.2.0^{\circ}\right)$ and at $3.5^{\circ}\left(1.5^{\circ}<\theta<5.5^{\circ}\right)$. Sample spectra obtained for ${ }^{56} \mathrm{Fe},{ }^{58} \mathrm{Ni}$ (from Ref. [10]), and ${ }^{60} \mathrm{Ni}$ are shown in 

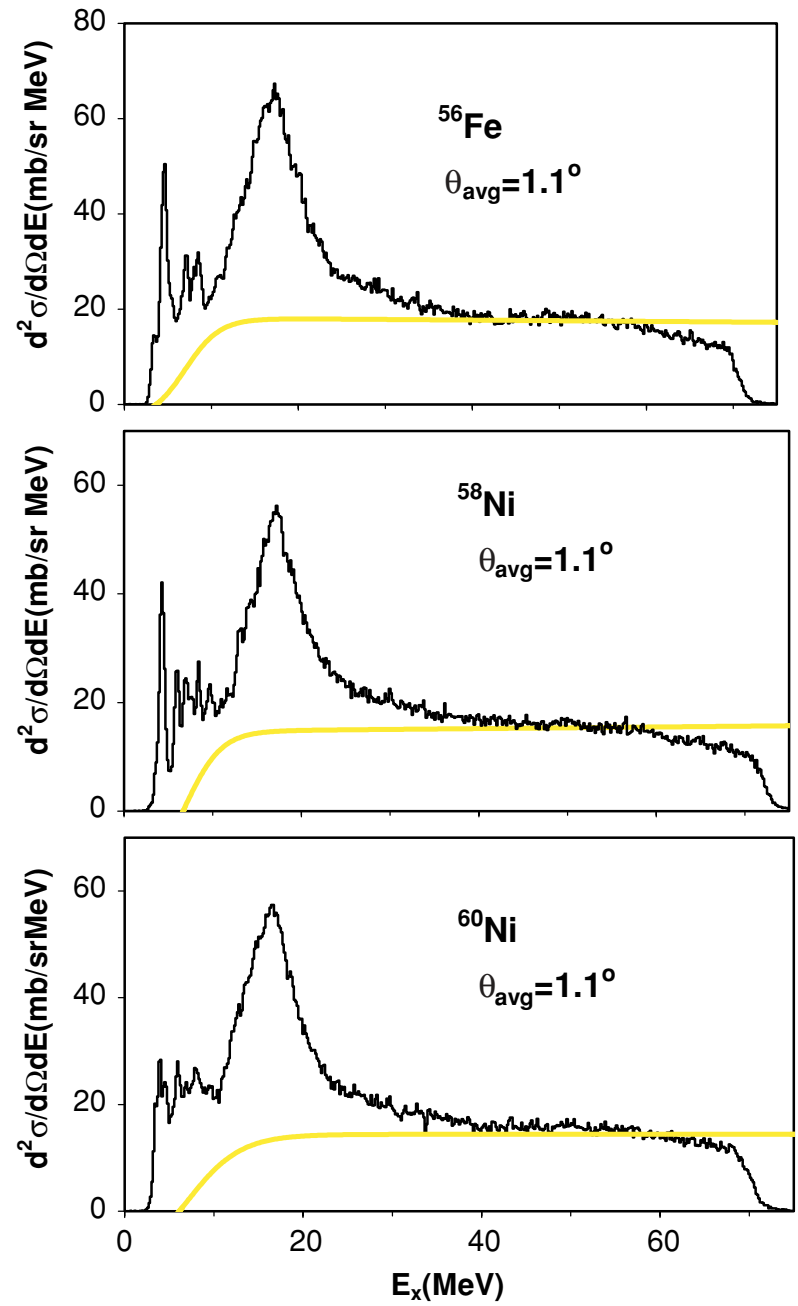

FIG. 1. (Color online) Inelastic $\alpha$ spectra obtained for ${ }^{56} \mathrm{Fe},{ }^{58} \mathrm{Ni}$, and ${ }^{60} \mathrm{Ni}$. The thick gray lines show the continuum chosen for analysis.

Fig. 1. The giant resonance peak can be seen extending up past $E_{x}=35 \mathrm{MeV}$. The spectrum was divided into a peak and a continuum, where the continuum was assumed to have the shape of a straight line at high excitation, joining onto
TABLE I. Folding model parameters for ${ }^{58} \mathrm{Ni}$ used in the DWBA calculations. $r_{P}$ and $r_{T}$ are the Coulomb radius parameters for projectile and target, respectively.

\begin{tabular}{lccccc}
\hline \hline$V(\mathrm{MeV})$ & $W(\mathrm{MeV})$ & $r_{i}$ & $a_{i}(\mathrm{fm})$ & $r_{P}$ & $r_{T}$ \\
\hline 41.19 & 40.39 & 0.821 & 0.974 & 1.336 & 1.256 \\
\hline \hline
\end{tabular}

a Fermi shape at low excitation to model particle threshold effects $[6,8]$. Samples of the continua used are shown in the figure.

\section{MULTIPOLE ANALYSIS}

Single-folding density-dependent distorted-wave Born approximation (DWBA) calculations (as described in Refs. $[6,8,15,16])$ were carried out. Radial moments for ${ }^{58} \mathrm{Ni}$ were obtained by numerical integration of the Fermi mass distribution with $c=4.08 \mathrm{fm}$ and $a=0.515 \mathrm{fm}$ [17]. Folding model parameters for ${ }^{58} \mathrm{Ni}$ were obtained from elasticscattering data of ${ }^{58} \mathrm{Ni}+\alpha$ at $240 \mathrm{MeV}$ extending from center-of-mass angles of $2^{\circ}-34^{\circ}$ and are listed in Table I. The fit obtained to ${ }^{58} \mathrm{Ni}$ elastic scattering with these parameters is shown in Fig. 2. DWBA calculations for the $1.454-\mathrm{MeV} 2^{+}$and $4.475-\mathrm{MeV}^{-}$states in ${ }^{58} \mathrm{Ni}$ using published electromagnetic $B(E L)$ values from Refs. [18,19] are shown superimposed on data obtained for those two states in Fig. 3. They are in good agreement with the experimental data. Elastic-scattering data were not available for ${ }^{56} \mathrm{Fe}$ or ${ }^{60} \mathrm{Ni}$, so the optical model parameters obtained for ${ }^{58} \mathrm{Ni}$ were used.

Calculations were performed with Fermi mass distributions $\left[\rho(r)=\rho_{0}\{1+\exp [(r-c) / a])^{-1}\right\}$ for ${ }^{56} \mathrm{Fe}$ and ${ }^{60} \mathrm{Ni}$ using $c=4.1198$ and 4.2328 , respectively, and $a=0.523 \mathrm{fm}$ for both nuclei [20]. The transition densities, sum rules, and DWBA calculations were discussed thoroughly in Refs. $[6,8]$ and, except for the isoscalar dipole, the same expressions and techniques were used in this work. The transition density for inelastic $\alpha$-particle excitation of the ISGDR given by Harakeh and Dieperink [21] (and described in Refs. [6,8]) is for only

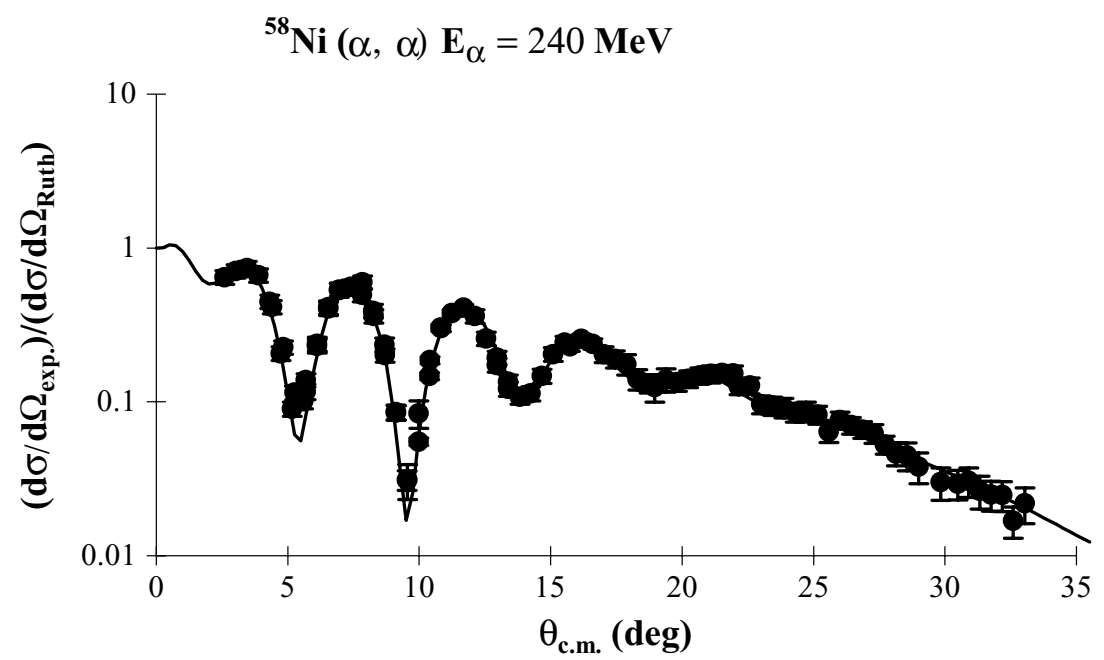

FIG. 2. Angular distribution of the ratio of the differential cross section for elastic scattering to Rutherford scattering for $240-\mathrm{MeV} \alpha$ particles from ${ }^{58} \mathrm{Ni}$ is plotted versus average center-ofmass angle. The error bars include uncertainty from statistical as well as systematic error. The solid line shows an optical model calculation with the parameters listed in Table I. 

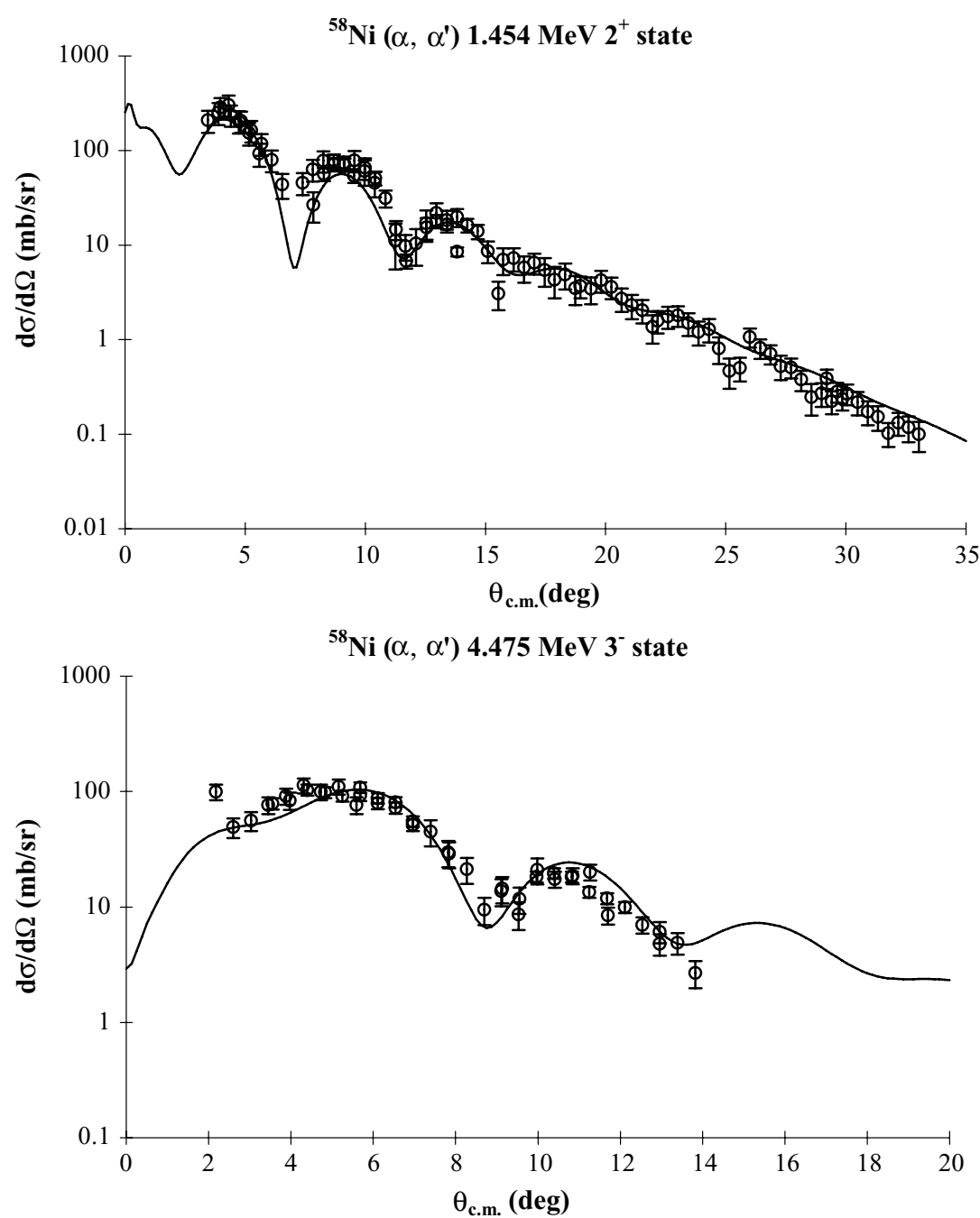

FIG. 3. (Top) Angular distribution of the differential cross section for inelastic $\alpha$ scattering to the $1.454-\mathrm{MeV} 2^{+}$state in ${ }^{58} \mathrm{Ni}$ plotted versus average center-of-mass angle. The solid line shows an $L=2$ DWBA calculation using the $B(E 2)$ value from electron scattering [18]. (Bottom) Angular distribution of the differential cross section for inelastic $\alpha$ scattering to the $4.475 \mathrm{MeV} 3^{-}$state in ${ }^{58} \mathrm{Ni}$ plotted versus average center-of-mass angle. The solid line shows an $L=3$ DWBA calculation using the $B(E 3)$ value from electron scattering [19]. one magnetic substate, so that the transition density given in Ref. [21] must be multiplied by $\sqrt{3}$ in the DWBA calculation.

The multipole components of the giant resonance peak were obtained $[6,8]$ by dividing the peak into multiple regions (bins) by excitation energy and then comparing the angular distributions obtained for each of these bins to DWBA calculations for isoscalar $L=0,1,2$, and 3 transitions. The uncertainty from the multipole fits was determined for each multipole by incrementing (or decrementing) that strength and then adjusting the strengths of the other multipoles to minimize total $\chi^{2}$. This continued until the new $\chi^{2}$ was one unit larger than the total $\chi^{2}$ obtained for the best fit. A sample of the angular distributions obtained for the giant resonance peak and continuum are shown for ${ }^{56} \mathrm{Fe},{ }^{58} \mathrm{Ni}$, and ${ }^{60} \mathrm{Ni}$ in Figs. 4-6, respectively, and the fits obtained are shown superimposed on the data.

Several analyses were carried out to assess the effects of different choices of the continuum on the resulting multipole distribution, as described in Ref. [4], where the continuum was systematically varied and the data were reanalyzed. Typical choices of continuum are shown in gray and black lines in Fig. 7. The strength distributions obtained from these analyses and from those obtained with the continua shown in Fig. 1 were then averaged, and errors were calculated by adding the errors obtained from the multipole fits in quadrature to the standard deviations between the different fits. The (isoscalar) $E 0, E 1$, $E 2$, and E3 distributions obtained are shown in Figs. 8-10, and the energy moments and sum-rule strengths obtained are summarized in Tables II, III, and IV. A single Gaussian was fit to the $E 0$ and $E 2$ distributions and two Gaussians were fit to the $E 1$ distributions. These are shown in Figs. 8 to 10 and the parameters obtained are listed in Tables II to IV.

\section{DISCUSSION}

For E0 energy-weighted sum rule (EWSR) strength, $98_{-10}^{+14} \%, 85_{-10}^{+13} \%$, and $82_{-11}^{+13} \%$ were located in ${ }^{56} \mathrm{Fe},{ }^{58} \mathrm{Ni}$, and ${ }^{60} \mathrm{Ni}$, respectively. Almost all of the E0 EWSR strength was found below $35 \mathrm{MeV}$ in these nuclei. The shape of the strength distributions are asymmetric, following a Gaussian shape on the low excitation side but tailing on the high excitation side. The GMR distribution obtained for ${ }^{58} \mathrm{Ni}$ is in excellent agreement with the previous analysis [10], as can be seen in Fig. 9.

More than $70 \%$ of the E2 EWSR was located in all three nuclei, in an almost Gaussian peak between $E_{x}=10$ and $25 \mathrm{MeV}$. The centroid and root-mean-square (rms) width of 

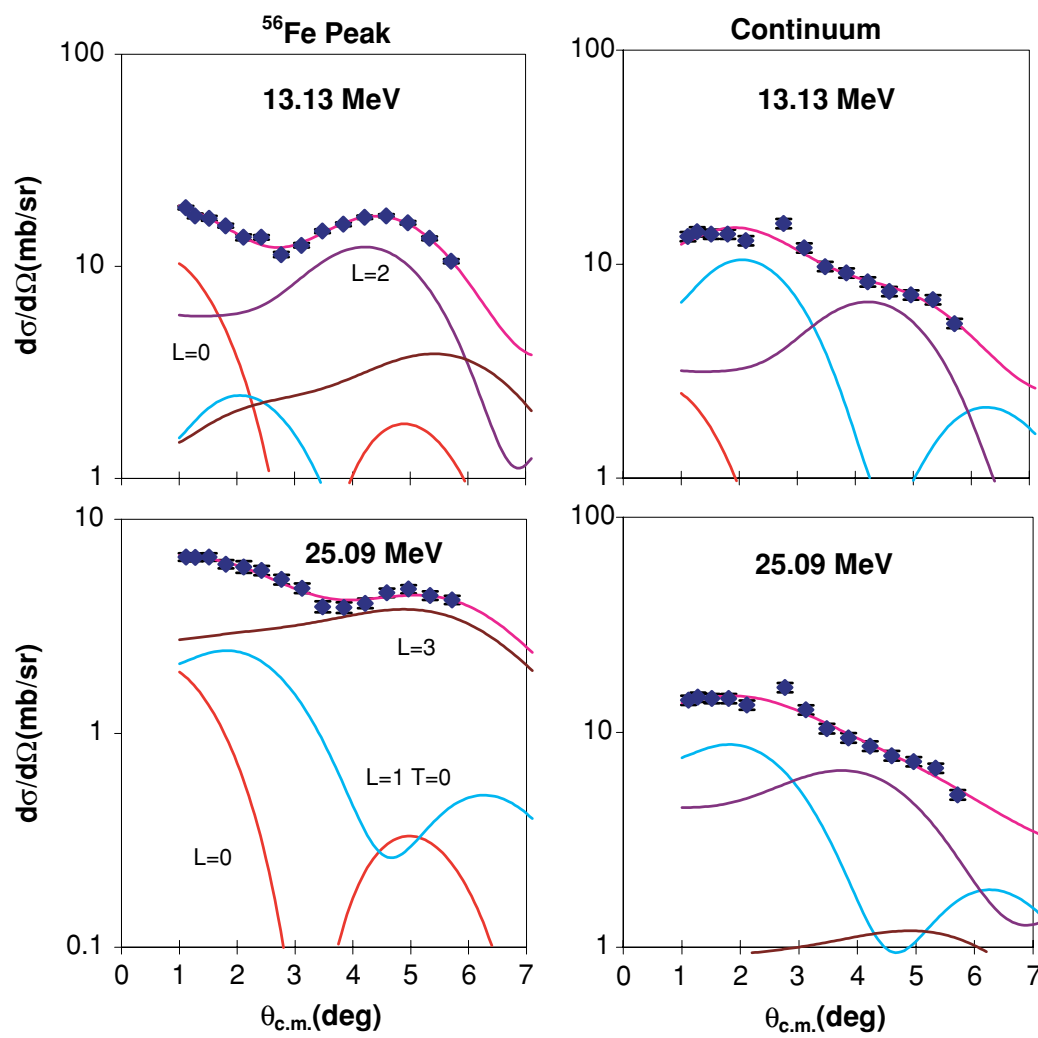

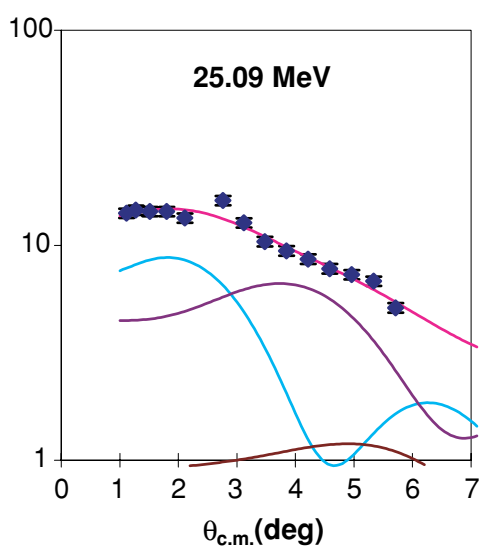

FIG. 4. (Color online) The angular distributions of the ${ }^{56} \mathrm{Fe}$ cross sections for two excitation ranges of the GR peak and the continuum. Each bin is $790 \mathrm{keV}$ wide and the average energies for each bin are shown. The lines through the data points indicated the multipole fits. Contributions of each multipole are shown. The statistical errors are smaller than the data points. the E2 strength in ${ }^{58} \mathrm{Ni}$ is in good agreement with Ref. [10]; however, the total strength located $(82 \pm 10 \%)$ is somewhat smaller (113 $\pm 15 \%$ in Ref. [10]). In Ref. [10] it was pointed out that the $E 2$ strength was sensitive to the choice of continuum and in Ref. [10], the authors explicitly did not assign an uncertainty because of the choice of continuum for the $E 2$ strength. The $E 2$ strength distributions reported here for ${ }^{58} \mathrm{Ni}$ and ${ }^{60} \mathrm{Ni}$ are in excellent agreement with the results
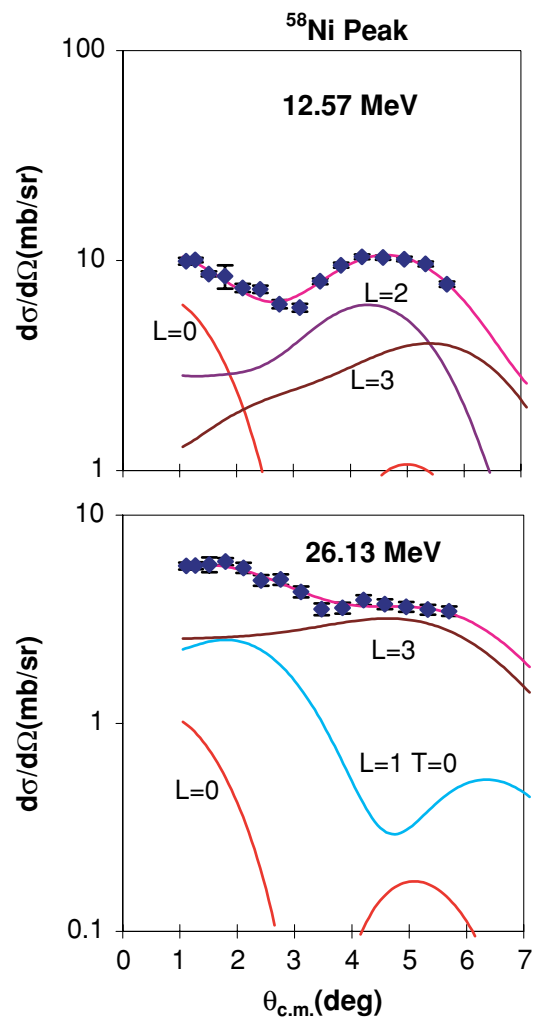
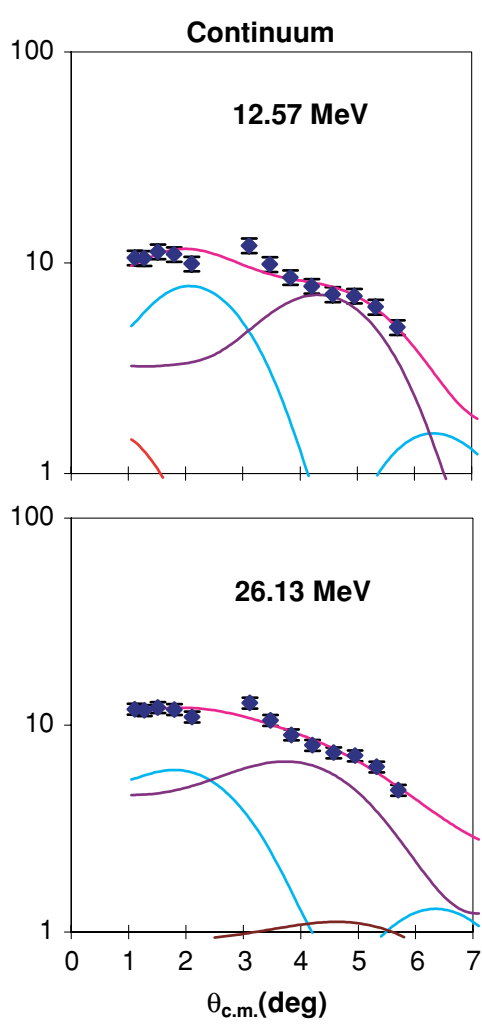

FIG. 5. (Color online) The angular distributions of the ${ }^{58} \mathrm{Ni}$ cross sections for two excitation ranges of the GR peak and the continuum. See the legend to Fig. 4. 

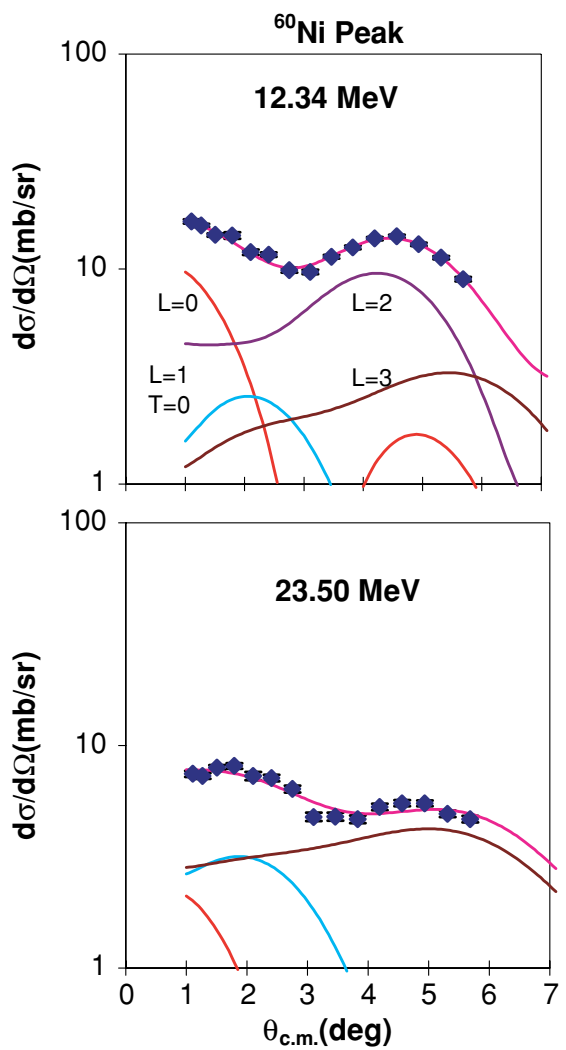
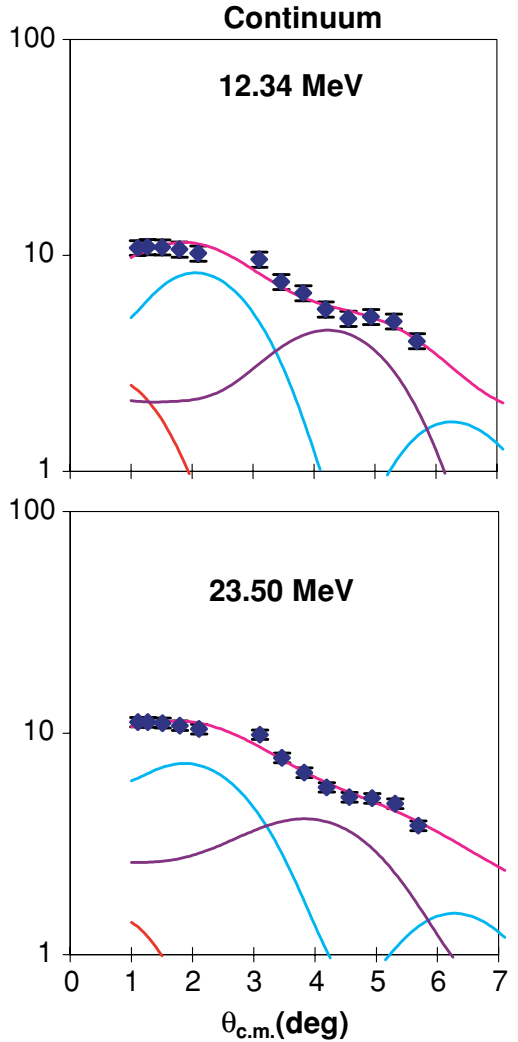

FIG. 6. (Color online) The angular distributions of the ${ }^{60} \mathrm{Ni}$ cross sections for two excitation ranges of the GR peak and the continuum. See the legend to Fig. 4. obtained by Youngblood et al. [22] using 129-MeV $\alpha$-particle scattering.

There have been no previous reports of ISGDR strength in medium-weight nuclei. The isoscalar dipole resonance is known to consist of two components [23-25] in heavier nuclei and the same feature can be seen in Figs. 8-10 for these nuclei. Between 56 and $72 \%$ of the E1 EWSR strength was located, and unlike in heavy nuclei, the high-excitation component dominates in the distribution with strength extended to and probably above $E_{x}=40 \mathrm{MeV}$. It is difficult to extract the $E 1$

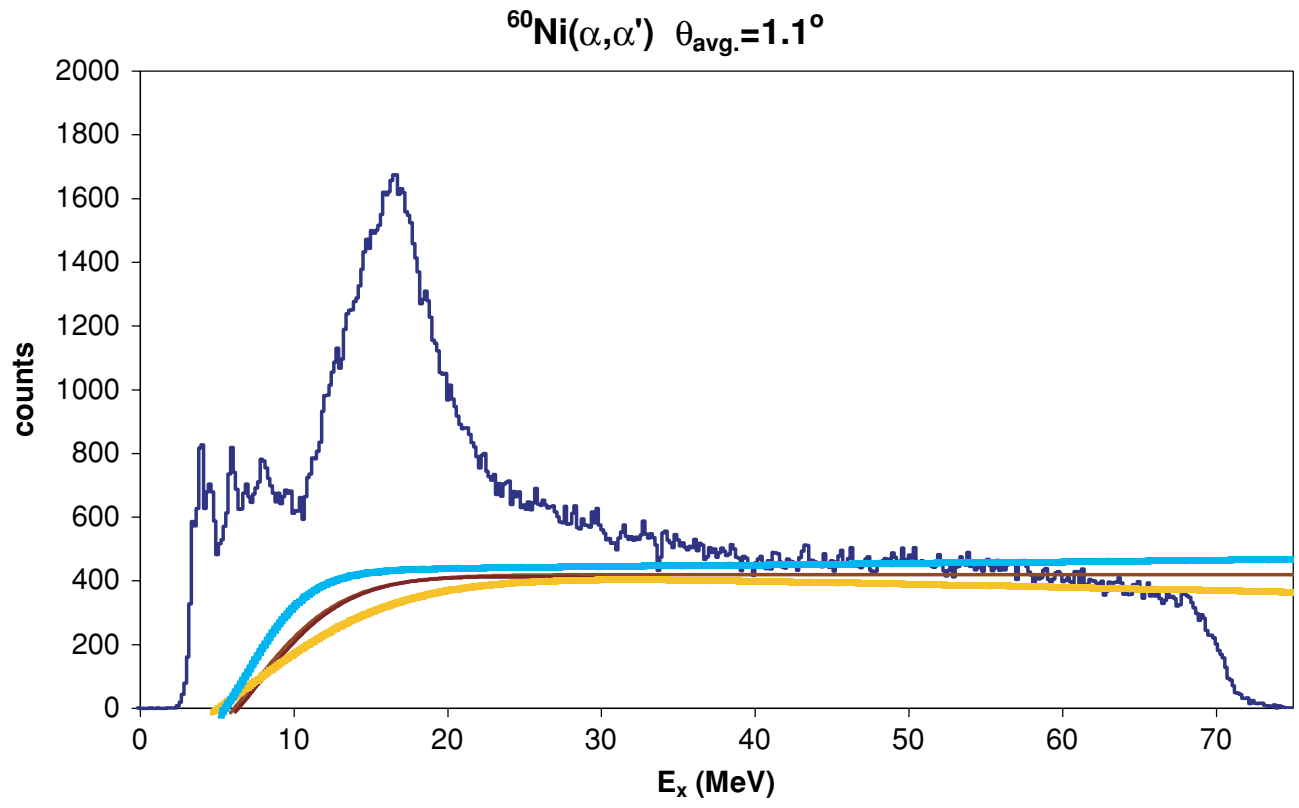

FIG. 7. (Color online) Inelastic $\alpha$ spectrum obtained for ${ }^{60} \mathrm{Ni}$ at $\theta_{\text {avg. }}=1.1^{\circ}$. The thick gray and black lines show the range of continuum choices used to check the effects on the resulting multipole distributions. See text. 

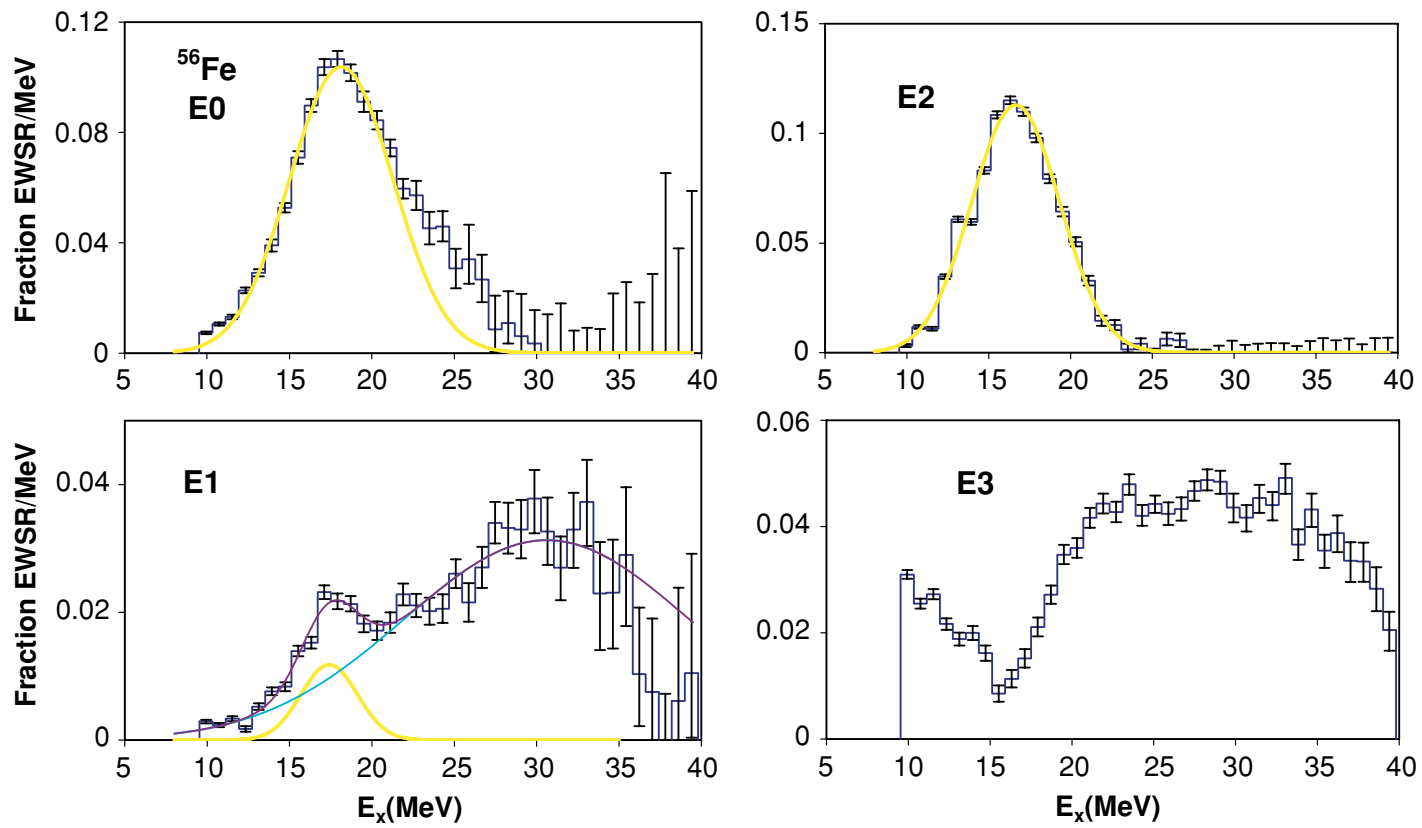

FIG. 8. (Color online) Strength distributions obtained for ${ }^{56} \mathrm{Fe}$ are shown by the histograms. Error bars represent the uncertainty because of the fitting of the angular distributions and different choices of the continuum, as described in the text. The E3 distributions includes all $L \geqslant 3$ strength. See text. The smooth lines show Gaussian fits.

strength reliably above $30 \mathrm{MeV}$ because of small ISGDR cross sections and the small peak-to-continuum ratio. The extracted $E 1$ strength becomes very sensitive to the choice of continuum and this is reflected in the large errors above $30 \mathrm{MeV}$.
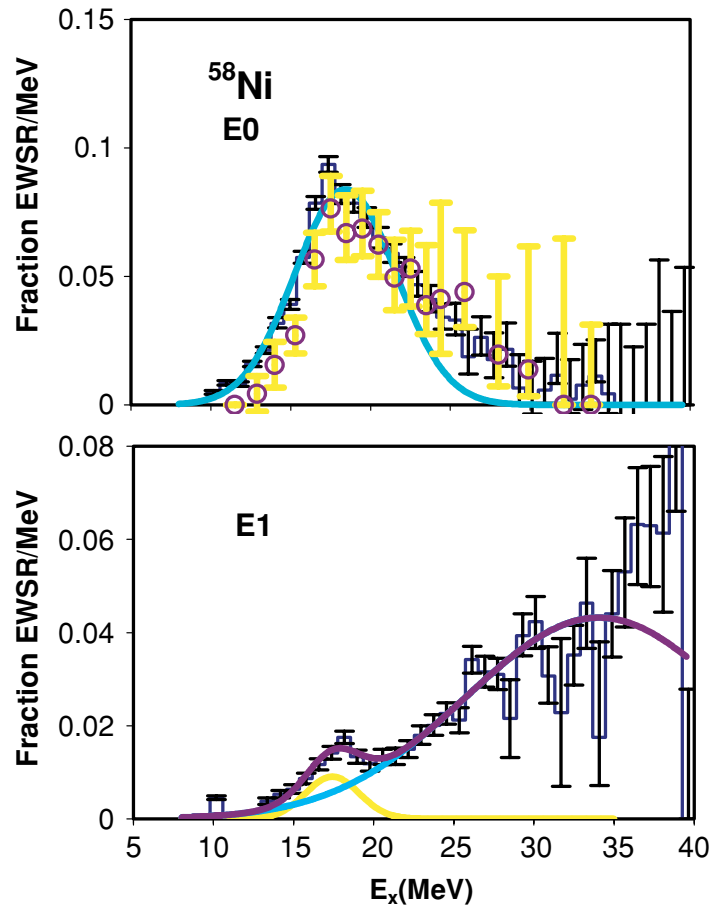

Because of the limited angular range of the data, $E 3$ strength could not reliably be separated from $E 4$ and higher, so the highest multipole included in the fits was $E 3$. Thus the "E3" distributions shown in Figs. 8-10 are actually the sum of all
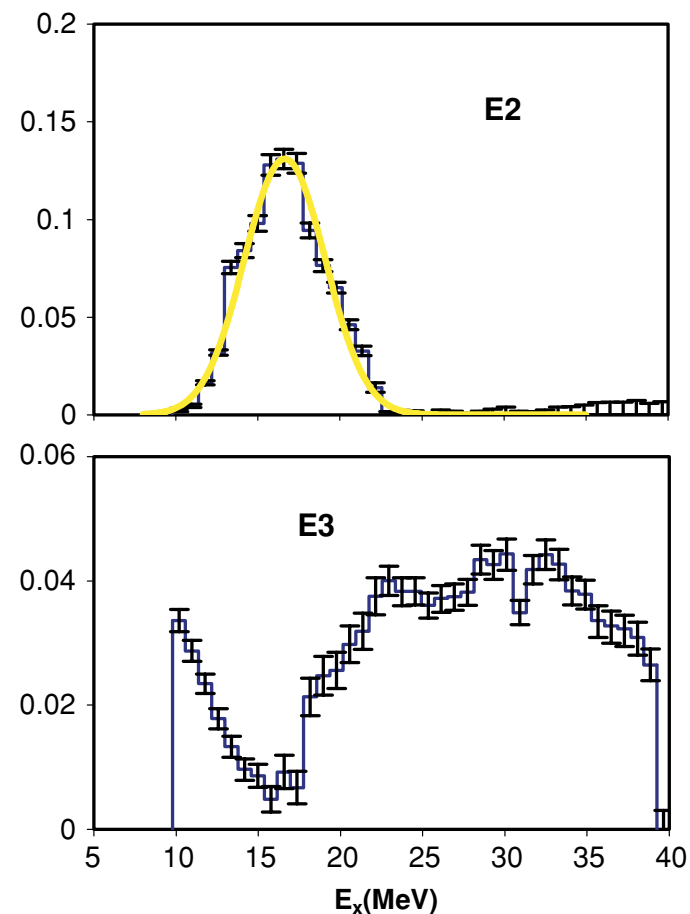

FIG. 9. (Color online) Strength distributions obtained for ${ }^{58} \mathrm{Ni}$ are shown by the histograms. The open circles indicated the $E 0$ strength distribution from Ref. [10]. See the legend to Fig. 8. 

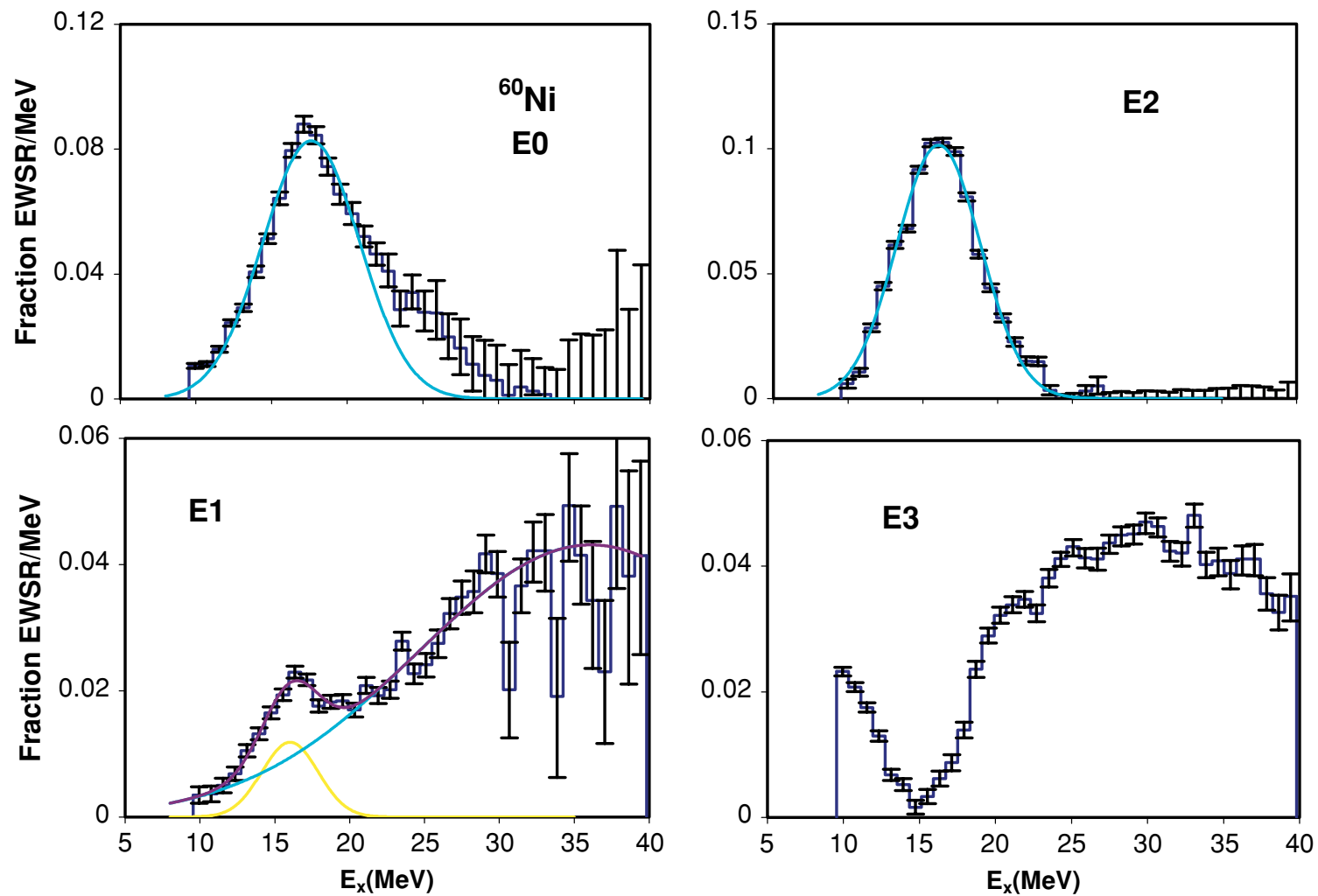

FIG. 10. (Color online) Strength distributions obtained for ${ }^{60} \mathrm{Ni}$ are shown by the histograms. See the legend to Fig. 8 .

multipoles $L \geqslant 3$. E3 strength is divided into two components $[26,27]$, the $1 \hbar \omega$ low-energy octupole resonance (LEOR) and the $3 \hbar \omega$ high-energy octupole resonance (HEOR). This feature is apparent in the "E3" distributions in Figs. 8-10. There is a large component starting from $E_{x} \sim 15 \mathrm{MeV}$ extending up to (and possibly past) $40 \mathrm{MeV}$, which, if it were entirely $E 3$ strength, would correspond to 86,76 , and $81 \%$ of the E3 EWSR in ${ }^{56} \mathrm{Fe},{ }^{58} \mathrm{Ni}$, and ${ }^{60} \mathrm{Ni}$, respectively. This is not too different from the $75 \%$ of the $E 3$ strength that should be in the $3 \hbar \omega$ component, suggesting the contributions to the peak from higher multipoles may be small.

Kamerdzhiev et al. [28] have carried out microscopic calculations for ${ }^{40} \mathrm{Ca}$ and ${ }^{58} \mathrm{Ni}$, using a microscopic nuclear structure model that takes into account known mechanisms of giant resonance damping, including the spreading width caused by more complex $1 \mathrm{p} 1 \mathrm{~h}$ coupled to phonon configurations and the escape width because of inclusion of the single-particle continuum. Their calculations were focused on

TABLE II. Parameters obtained for isoscalar multipoles in ${ }^{56} \mathrm{Fe}$.

\begin{tabular}{lrrrr}
\hline \hline & \multicolumn{5}{c}{ Moments } \\
\cline { 2 - 5 } & \multicolumn{1}{c}{$E 0$} & \multicolumn{1}{c}{$E 1$} & $E 2$ & $E 3$ and higher \\
\hline$m_{1}$ (Frac. EWSR) & $0.98_{-0.10}^{+0.14}$ & $0.56_{-0.15}^{+0.20}$ & $0.77 \pm 0.10$ & $1.00 \pm 0.10$ \\
$m_{1} / m_{0}(\mathrm{MeV})$ & $18.35_{-0.19}^{+0.33}$ & $24.37_{-0.31}^{+0.49}$ & $16.35_{-0.10}^{+0.19}$ & $22.76 \pm 0.26$ \\
$\mathrm{rms}$ width $(\mathrm{MeV})$ & $4.01_{-0.18}^{+1.11}$ & $6.67_{-0.25}^{+0.28}$ & $2.87 \pm 0.12$ & $8.14 \pm 0.17$ \\
$\left(m_{3} / m_{1}\right)^{1 / 2}(\mathrm{MeV})$ & $19.57_{-0.16}^{+0.73}$ & & & \\
$\left(m_{1} / m_{-1}\right)^{1 / 2}(\mathrm{MeV})$ & $17.92_{-0.15}^{+0.26}$ & & &
\end{tabular}

Gaussian fits

\begin{tabular}{lcccc}
\cline { 2 - 4 } & $E 0$ & $E 1$ peak 1 & $E 1$ peak 2 & $E 2$ \\
\hline Centroids (MeV) & $18.14_{-0.15}^{+0.14}$ & $17.41 \pm 0.20$ & $30.61 \pm 0.29$ & $16.65 \pm 0.11$ \\
FWHM (MeV) & $7.40 \pm 0.13$ & $4.03_{-0.26}^{+0.28}$ & $20.31_{-0.40}^{+0.39}$ & $6.35_{-0.11}^{+0.16}$ \\
Frac. EWSR & $0.82_{-0.08}^{+0.10}$ & $0.05 \pm 0.02$ & $0.67 \pm 0.10$ & $0.76 \pm 0.10$ \\
\hline \hline
\end{tabular}


TABLE III. Parameters obtained for isoscalar multipoles in ${ }^{58} \mathrm{Ni}$.

\begin{tabular}{lrrrr}
\hline \hline & \multicolumn{5}{c}{ Moments } \\
\cline { 2 - 5 } & \multicolumn{1}{c}{$E 0$} & \multicolumn{1}{c}{$E 1$} & $E 2$ & $E 3$ and higher \\
\hline$m_{1}($ Frac. EWSR $)$ & $0.85_{-0.10}^{+0.13}$ & $0.68_{-0.15}^{+0.20}$ & $0.82 \pm 0.10$ & $0.87 \pm 0.10$ \\
$m_{1} / m_{0}(\mathrm{MeV})$ & $19.20_{-0.19}^{+0.44}$ & $27.78_{-0.30}^{+0.47}$ & $16.31_{-0.10}^{+0.17}$ & $23.20 \pm 0.30$ \\
rms width $(\mathrm{MeV})$ & $4.89_{-0.31}^{+1.05}$ & $6.96_{-0.25}^{+0.30}$ & $2.45 \pm 0.10$ & $8.24 \pm 0.12$ \\
$\left(m_{3} / m_{1}\right)^{1 / 2}(\mathrm{MeV})$ & $20.81_{-0.28}^{+0.90}$ & & & \\
$\left(m_{1} / m_{-1}\right)^{1 / 2}(\mathrm{MeV})$ & $18.70_{-0.17}^{+0.34}$ & & &
\end{tabular}

Gaussian fits

\begin{tabular}{lcccc}
\cline { 2 - 4 } & $E 0$ & $E 1$ peak 1 & $E 1$ peak 2 & $E 2$ \\
\hline Centroids (MeV) & $18.43 \pm 0.15$ & $17.42 \pm 0.25$ & $34.06 \pm 0.30$ & $16.64 \pm 0.12$ \\
FWHM (MeV) & $7.41 \pm 0.13$ & $3.94_{-0.34}^{+0.36}$ & $19.52_{-0.40}^{+0.41}$ & $5.81_{-0.11}^{+0.16}$ \\
Frac. EWSR & $0.82_{-0.09}^{+0.11}$ & $0.04 \pm 0.02$ & $0.86 \pm 0.12$ & $0.81 \pm 0.10$ \\
\hline \hline
\end{tabular}

reproducing isoscalar multipole strength distributions rather than obtaining nuclear matter compressibility. They calculate expected cross sections as a function of $E_{x}$ for $240-\mathrm{MeV}$ inelastic $\alpha$-particle scattering, including $L=0-4$, and get excellent agreement with our ${ }^{58} \mathrm{Ni}$ cross section [10]. Their predicted multipole distributions are compared with our results in Fig. 11. Their E0 distribution is in excellent agreement with the experimental distribution; however, their isoscalar $E 1, E 2$, and $E 3$ distributions are not as consistent with our experimental results. Their predicted $E 2$ distribution differs significantly from the experimental distribution. They show strength corresponding to about half of the E2 EWSR between $E_{x}=10-25 \mathrm{MeV}$, with the rest extending up to $E_{x}=45 \mathrm{MeV}$, whereas the measured E2 distribution is nearly Gaussian, centered at $E_{x}=16.3 \mathrm{MeV}$ and contains $82 \pm 10 \%$ of the $E 2$ EWSR. We see no $E 2$ strength above $E_{x}=25 \mathrm{MeV}$. Though we cannot separate $E 3$ strength from higher multipoles, we see little strength in the vicinity of $E_{x}=16 \mathrm{MeV}$, where they show a strong peak, though the higher peak around $E_{x}=$
$30 \mathrm{MeV}$ is in reasonable agreement with our data. Although the experimental errors are large, the higher excitation peak predicted in the isoscalar $E 1$ strength distribution is about $10 \mathrm{MeV}$ below the observed peak.

There are no specific microscopic calculations for $E 0$ strength in ${ }^{56} \mathrm{Fe}$ and ${ }^{60} \mathrm{Ni}$; however, Nayak et al. [29] have carried out Hartree-Fock random-phase (HF-RPA) calculations with several Skyrme or Skyrme-like interactions and parameterized the results in terms of the Leptodermous expansion. Chossy and Stocker [30] have carried out a similar parametrization for several relativistic mean-field (RMF) parameter sets. E0 energies calculated with relativistic and nonrelativistic interactions are compared to the experimental energies $\left(m_{3} / m_{1}\right)^{1 / 2}\left(m_{k}=\sum\left(E_{n}-E_{0}\right)^{k}\left|\left\langle 0\left|r^{2}\right| n\right\rangle\right|^{2}\right)$ for ${ }^{56} \mathrm{Fe}$, ${ }^{58} \mathrm{Ni}$, and ${ }^{60} \mathrm{Ni}$ and are shown together with ${ }^{90} \mathrm{Zr}$ and ${ }^{40} \mathrm{Ca}$ data in Fig. 12. The GMR energies in ${ }^{56} \mathrm{Fe}$ and ${ }^{60} \mathrm{Ni}$ are in excellent agreement with calculation for interactions for which $K_{\mathrm{NM}} \sim 211-217 \mathrm{MeV}$. This is also consistent with the results in the study of isotopic dependence of monopole energies

TABLE IV. Parameters obtained for isoscalar multipoles in ${ }^{60} \mathrm{Ni}$.

\begin{tabular}{lrrrr}
\hline \hline & \multicolumn{5}{c}{ Moments } \\
\cline { 2 - 5 } & \multicolumn{1}{c}{$E 0$} & \multicolumn{1}{c}{$E 1$} & $E 2$ & E3 and higher \\
\hline$m_{1}($ Frac. EWSR $)$ & $0.82_{-0.11}^{+0.13}$ & $0.72 \pm 0.17$ & $0.71 \pm 0.10$ & $0.88 \pm 0.10$ \\
$m_{1} / m_{0}(\mathrm{MeV})$ & $18.04_{-0.23}^{+0.35}$ & $24.93 \pm 0.46$ & $15.84_{-0.10}^{+0.18}$ & $24.40 \pm 0.26$ \\
$\mathrm{rms}$ width $(\mathrm{MeV})$ & $4.50_{-0.22}^{+0.97}$ & $7.65 \pm 0.27$ & $2.92 \pm 0.10$ & $7.65 \pm 0.18$ \\
$\left(m_{3} / m_{1}\right)^{1 / 2}(\mathrm{MeV})$ & $19.54_{-0.23}^{+0.78}$ & & & \\
$\left(m_{1} / m_{-1}\right)^{1 / 2}(\mathrm{MeV})$ & $17.55_{-0.17}^{+0.27}$ & & &
\end{tabular}

Gaussian fits

\begin{tabular}{lcccc}
\cline { 2 - 4 } & $E 0$ & $E 1$ peak 1 & $E 1$ peak 2 & $E 2$ \\
\hline Centroids (MeV) & $17.62 \pm 0.15$ & $16.01 \pm 0.20$ & $36.11_{-0.27}^{+0.29}$ & $16.05 \pm 0.12$ \\
FWHM (MeV) & $7.55 \pm 0.13$ & $4.41_{-0.22}^{+0.34}$ & $27.13_{-0.42}^{+0.43}$ & $6.61_{-0.11}^{+0.16}$ \\
Frac. EWSR & $0.67_{-0.09}^{+0.12}$ & $0.06 \pm 0.03$ & $1.20 \pm 0.16$ & $0.71 \pm 0.10$ \\
\hline \hline
\end{tabular}



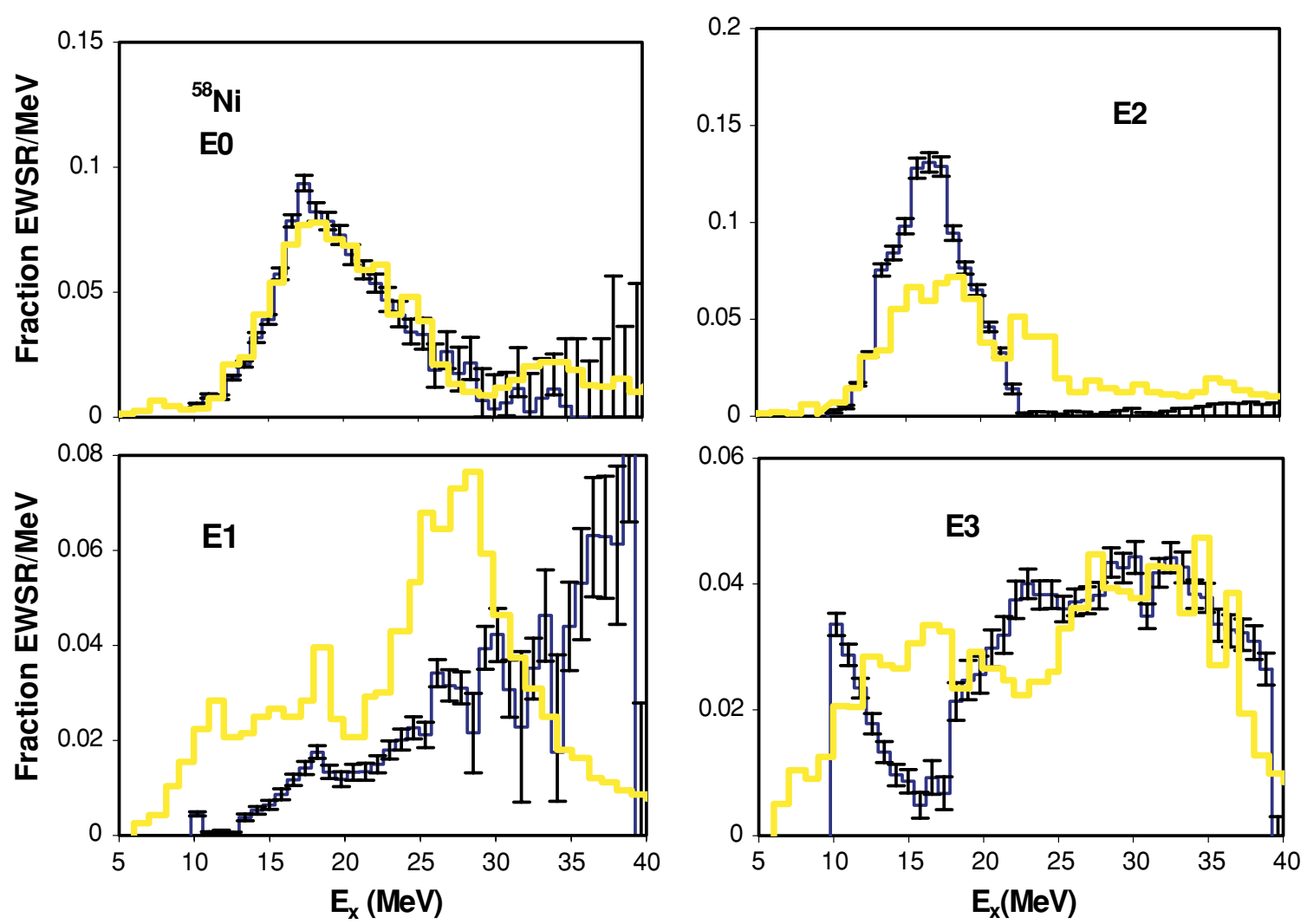

FIG. 11. (Color online) Strength distributions obtained for ${ }^{58} \mathrm{Ni}$ are shown by the histograms. See the legend to Fig. 8. The Gray lines are calculations from Kamerdzhiev et al. [28].

in ${ }^{112} \mathrm{Sn}$ and ${ }^{124} \mathrm{Sn}$ [31], as well as in ${ }^{110} \mathrm{Cd}$ and ${ }^{116} \mathrm{Cd}$ [9]. However, this is somewhat lower than $K_{\mathrm{NM}} \sim 231 \mathrm{MeV}$, suggested by energies for a number of other nuclei [3]. The nonrelativistic parametrization set $\mathrm{SkM}^{*}$ reproduce the GMR energy in ${ }^{40} \mathrm{Ca}$ [29], but not the relativistic parametrization set NL1 [30], and both of the calculations miss the energy of ${ }^{90} \mathrm{Zr}$. The experimental value of GMR energy in ${ }^{58} \mathrm{Ni}$ is more than 1.2 $\mathrm{MeV}$ higher than in ${ }^{56} \mathrm{Fe}$ and ${ }^{60} \mathrm{Ni}$, but both calculations fail to predict this feature. The ${ }^{56} \mathrm{Fe},{ }^{60} \mathrm{Ni}$, and ${ }^{58} \mathrm{Ni}$ data were all obtained in the same experimental run, so systematic errors (such as detector threshold effects, continuum choices, and energy calibrations) should be similar for these nuclei. The reason for the large energy difference between these adjacent nuclei is not clear, and microscopic calculations may be necessary to understand this effect. The GMR energy of ${ }^{58} \mathrm{Ni}$ is in good agreement with the relativistic NLC calculation for

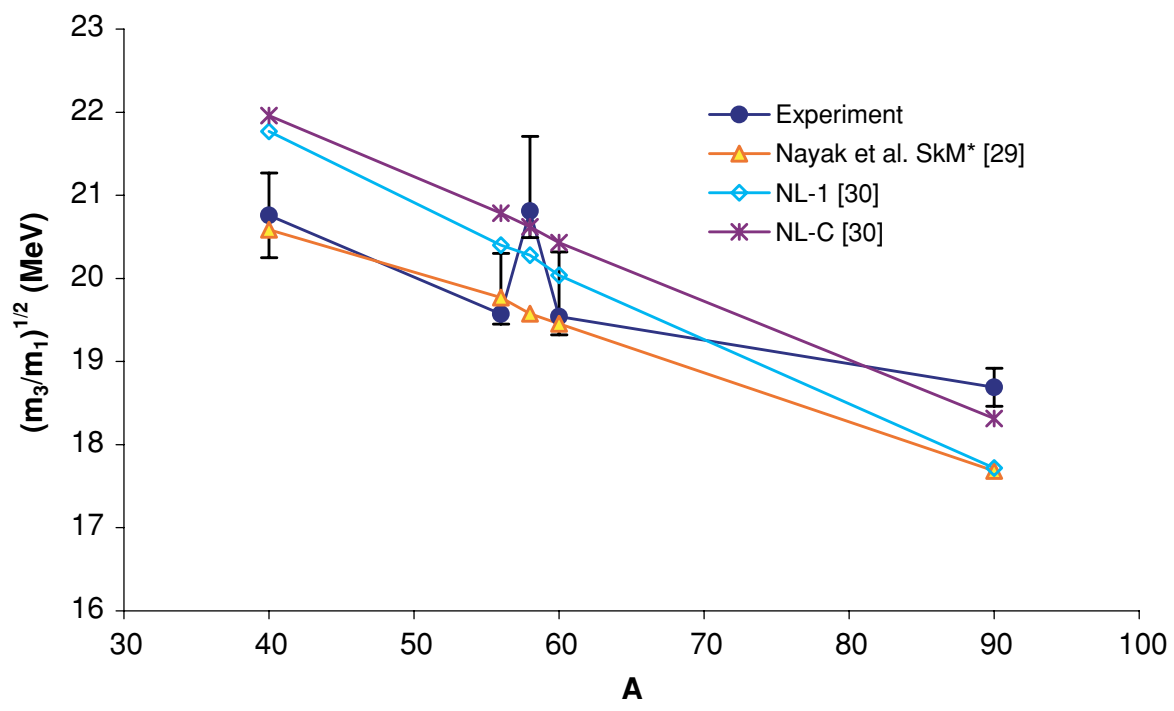

FIG. 12. (Color online) GMR energies calculated with the relativistic mean-field parametrization [30] and the nonrelativistic parametrizations [29] are compared to the experimental energies shown by solid circles. The error bars on the data include systematic errors. 
which $K_{\mathrm{NM}} \sim 225 \mathrm{MeV}$ [30]; however, this parametrization set does not reproduce the GMR energies of ${ }^{40} \mathrm{Ca}$ and ${ }^{90} \mathrm{Zr}$.

\section{CONCLUSION}

Most of the expected isoscalar $E 0$ and $E 2$ strength in ${ }^{56} \mathrm{Fe},{ }^{58} \mathrm{Ni}$, and ${ }^{60} \mathrm{Ni}$ has been identified below $E_{x}=40 \mathrm{MeV}$. Between 56 and $72 \%$ of the isoscalar $E 1$ strength has been located in these nuclei, and from the Gaussian fits to the experimental data 67,86 , and $120 \%$ of the isoscalar $E 1$ strength are suggested in ${ }^{56} \mathrm{Fe},{ }^{58} \mathrm{Ni}$, and ${ }^{60} \mathrm{Ni}$, respectively, in the high excitation component so that some of the missing strength in the experimental data likely lies above $E_{x}=$ $40 \mathrm{MeV}$. Our analysis did not separate multipoles with $L \geqslant 3$, so we can draw no definitive conclusion about $L=3$ strength distributions. The asymmetric shape of the GMR peak in ${ }^{56} \mathrm{Fe}$ and ${ }^{60} \mathrm{Ni}$ is consistent with that in ${ }^{58} \mathrm{Ni}[10]$ and clearly different from the symmetric peak in heavy nuclei. However, the giant quadrupole resonance peak is Gaussian in nuclei from ${ }^{40} \mathrm{Ca}$ [8] to ${ }^{208} \mathrm{~Pb}$. Predictions using relativistic and nonrelativistic (Skyrme or Skyrme-like) interactions with $K_{\mathrm{NM}} \sim$ 211-217 MeV result in energies in excellent agreement with the experimental GMR energies in ${ }^{56} \mathrm{Fe}$ and ${ }^{60} \mathrm{Ni}$. In ${ }^{58} \mathrm{Ni}$, the energy is more consistent with $K_{\mathrm{NM}} \sim 225 \mathrm{MeV}$. The relative energy of the GMR in ${ }^{58} \mathrm{Ni}$ and the other two nuclei is not consistent with the prediction using the Leptodermous expansion of HF-RPA results or RMF results and will likely require microscopic calculations to understand.

\section{ACKNOWLEDGMENTS}

This work was supported in part by the U.S. Department of Energy under grant DE-FG02-93ER40773 and by The Robert A. Welch Foundation.
[1] J. P. Blaizot, Phys. Rep. 64, 171 (1980).

[2] S. Stringari, Phys. Lett. B108, 232 (1982).

[3] D. H. Youngblood, H. L. Clark, and Y.-W. Lui, Phys. Rev. Lett. 82, 691 (1999).

[4] D. H. Youngblood, Y.-W. Lui, H. L. Clark, B. John, Y. Tokimoto, and X. Chen, Phys. Rev. C 69, 034315 (2004).

[5] B. John, Y. Tokimoto, Y.-W. Lui, H. L. Clark, X. Chen, and D. H. Youngblood, Phys. Rev. C 68, 014305 (2003).

[6] D. H. Youngblood, Y.-W. Lui, and H. L. Clark, Phys. Rev. C 65, 034302 (2002).

[7] Y.-W. Lui, H. L. Clark, and D. H. Youngblood, Phys. Rev. C 64, 064308 (2001).

[8] D. H. Youngblood, Y.-W. Lui, and H. L. Clark, Phys. Rev. C 63, 067301 (2001).

[9] Y.-W. Lui, D. H. Youngblood, Y. Tokimoto, H. L. Clark, and B. John, Phys. Rev. C 69, 034611 (2004).

[10] Y.-W. Lui, H. L. Clark, and D. H. Youngblood, Phys. Rev. C 61, 067307 (2000).

[11] D. H. Youngblood and Y.-W. Lui, Phys. Rev. C 44, 1878 (1991).

[12] M. Buenerd, J. Phys. (Paris) Colloq. 45, C4-115 (1984).

[13] S. Shlomo and D. H. Youngblood, Phys. Rev. C 47, 529 (1993).

[14] K. van der Borg, M. N. Harakeh, and A. van der Woude, Nucl. Phys. A365, 243 (1981).

[15] H. L. Clark, Y.-W. Lui, and D. H. Youngblood, Phys. Rev. C 57, 2887 (1998).

[16] G. R. Satchler and D. T. Khoa, Phys. Rev. C 55, 285 (1997).

[17] G. R. Satchler, Nucl. Phys. A472, 215 (1987).
[18] S. Raman, C. H. Malarkey, W. T. Milner, C. W. Nestor, and P. H. Stelson, At. Data Nucl. Data Tables 36, 1 (1987).

[19] R. H. Spear, At. Data Nucl. Data Tables 42, 55 (1989).

[20] G. Fricke, C. Bernhardt, K. Heilig, L. A. Schaller, L. Schellenberg, E. B. Shera, and C. W. De Jager, At. Data Nucl. Data Tables 60, 177 (1995).

[21] M. N. Harakeh and A. E. L. Dieperink, Phys. Rev. C 23, 2329 (1981).

[22] D. H. Youngblood, Y.-W. Lui, U. Garg, and R. J. Peterson, Phys. Rev. C 45, 2172 (1992).

[23] H. L. Clark, Y.-W. Lui, and D. H. Youngblood, Phys. Rev. C 63, 031301(R) (2001).

[24] G. Colo, N. van Giai, P. F. Bortignon, and M. R. Quaglia, Phys. Lett. B485, 362 (2000).

[25] D. Vretenar, A. Wandelt, and P. Ring, Phys. Lett. B487, 334 (2000).

[26] Aage Bohr and Ben R. Mottelson, Nuclear Structure (Benjamin, Reading, MA, 1975), Vol. II.

[27] J. M. Moss, D. H. Youngblood, C. M. Rozsa, D. R. Brown, and J. D. Bronson, Phys. Rev. Lett. 37, 816 (1976).

[28] S. Kamerdzhiev, J. Speth, and G. Tertychny, Eur. Phys. J. A 7, 483 (2000).

[29] R. C. Nayak, J. M. Pearson, M. Farine, P. Gleissl, and M. Brack, Nucl. Phys. A516, 62 (1990).

[30] T. v. Chossy and W. Stocker, Phys. Rev. C 56, 2518 (1997).

[31] Y.-W. Lui, D. H. Youngblood, Y. Tokimoto, H. L. Clark, and B. John, Phys. Rev. C 70, 014307 (2004). 